
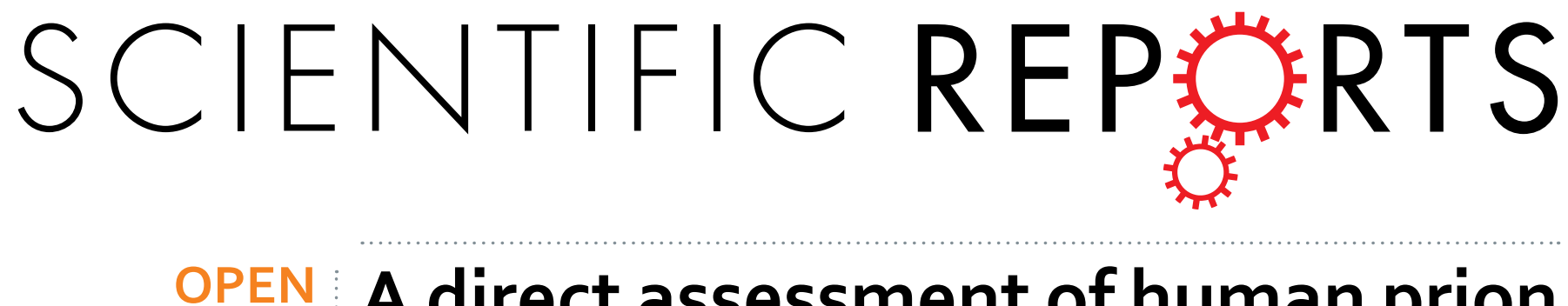

\title{
A direct assessment of human prion adhered to steel wire using real- time quaking-induced conversion
}

Received: 05 January 2016

Accepted: 08 April 2016

Published: 26 April 2016
Tsuyoshi Mori ${ }^{1, \#}$, Ryuichiro Atarashi ${ }^{1, \#}$, Kana Furukawa ${ }^{1}$, Hanae Takatsuki ${ }^{1}$, Katsuya Satoh ${ }^{2}$, Kazunori Sano ${ }^{3}$, Takehiro Nakagaki ${ }^{1}$, Daisuke Ishibashi ${ }^{1}$, Kazuko Ichimiya ${ }^{4}$, Masahisa Hamada ${ }^{4}$, Takehisa Nakayama ${ }^{4}$ \& Noriyuki Nishida ${ }^{1}$

Accidental transmission of prions during neurosurgery has been reported as a consequence of re-using contaminated surgical instruments. Several decontamination methods have been studied using the 263K-hamster prion; however, no studies have directly evaluated human prions. A newly developed in vitro amplification system, designated real-time quaking-induced conversion (RT-QuIC), has allowed the activity of abnormal prion proteins to be assessed within a few days. RT-QuIC using human recombinant prion protein (PrP) showed high sensitivity for prions as the detection limit of our assay was estimated as $0.12 \mathrm{fg}$ of active prions. We applied this method to detect human prion activity on stainless steel wire. When we put wires contaminated with human Creutzfeldt-Jakob disease brain tissue directly into the test tube, typical PrP-amyloid formation was observed within 48 hours, and we could detect the activity of prions at $50 \%$ seeding dose on the wire from $10^{2.8}$ to $10^{5.8} \mathrm{SD}_{50}$. Using this method, we also confirmed that the seeding activities on the wire were removed following treatment with $\mathrm{NaOH}$. As seeding activity closely correlated with the infectivity of prions using the bioassay, this wire-QuIC assay will be useful for the direct evaluation of decontamination methods for human prions.

Prion diseases, also known as transmissible spongiform encephalopathies (TSEs), such as bovine spongiform encephalopathy in cattle, scrapie in sheep and Creutzfeldt-Jakob disease (CJD) in humans, are fatal neurodegenerative disorders. At present, there is no effective therapy available for the diseases ${ }^{1}$. A host encoded normal prion protein, $\mathrm{PrP}^{\mathrm{C} 2}$, is required for susceptibility to prion infection ${ }^{3-5}$, and a hallmark of prion diseases is the accumulation of misfolded forms of $\operatorname{PrP}, \mathrm{PrP}^{\mathrm{Sc}}$. This amyloidogenic abnormally folded protein can be infectious. In human prion diseases, most cases (80\%) are categorised as sporadic and approximately $15 \%$ of cases are a genetic form carrying a mutation in the prion protein gene PRNP. Less than $1 \%$ are caused by accidental transmission $^{6}$. The possible iatrogenic transmission of such diseases was originally pointed out by Gajdusek in 1970s when the transmissibility of Kuru and CJD was evidenced ${ }^{7}$. In Japan, more than 140 cases of iatrogenic CJD have been identified following dura mater grafting from 1985 until now ${ }^{8}$. Accidental iatrogenic transmission of sporadic CJD (sCJD) has only occurred during neurosurgical procedures ${ }^{9}$. Until now, no cases of iatrogenic transmission following general surgery of nervous tissue or through endoscopic procedures have been reported ${ }^{9}$. Furthermore, experimental transmission studies using non-human primates demonstrated that bodily secretions are not infectious and that potential prion contamination of endoscopic instruments is not sufficient to cause human-to-human transmission ${ }^{10}$. In the case of CJD, infectivity is limited to the central nervous system; however, more recently many peripheral tissues from patients with valiant CJD have been shown to be infectious, and $\mathrm{PrP}^{\mathrm{Sc}}$ has also been detected in lymphoid organs such as the thymus, tonsils and spleen ${ }^{11}$. Moreover, $\operatorname{PrP}^{\mathrm{Sc}}$ has been detected in muscle, but no evidence of iatrogenic transmission was reported, suggesting a risk of iatrogenic transmission via contaminated surgical instruments ${ }^{12}$.

${ }^{1}$ Department of Molecular Microbiology and Immunology, Nagasaki University Graduate School of Biomedical Sciences, 1-12-4 Sakamoto, Nagasaki 852-8523, Japan. ²Department of Locomotive Rehabilitation Science, Nagasaki University Graduate School of Biomedical Sciences, 1-7-1 Sakamoto, Nagasaki 852-8523, Japan. ${ }^{3}$ Department of Physiology and Pharmacology, Faculty of Pharmaceutical Sciences, Fukuoka University, 8-19-1 Nanakuma, Jonan-ku, Fukuoka 814-0180, Japan. ${ }^{4}$ Kripton Co., Ltd., Dai 12 Daitetsu BIdg. 7F., 4-3-12 Yotsuya, Shinjuku-ku, Tokyo 160-0004, Japan. \#Present address: Department of Infectious Diseases, Faculty of Medicine, University of Miyazaki, 5200 Kihara, Kiyotake, Miyazaki 889-1692, Japan. Correspondence and requests for materials should be addressed toT.M. (email: morit@nagasaki-u.ac.jp) 


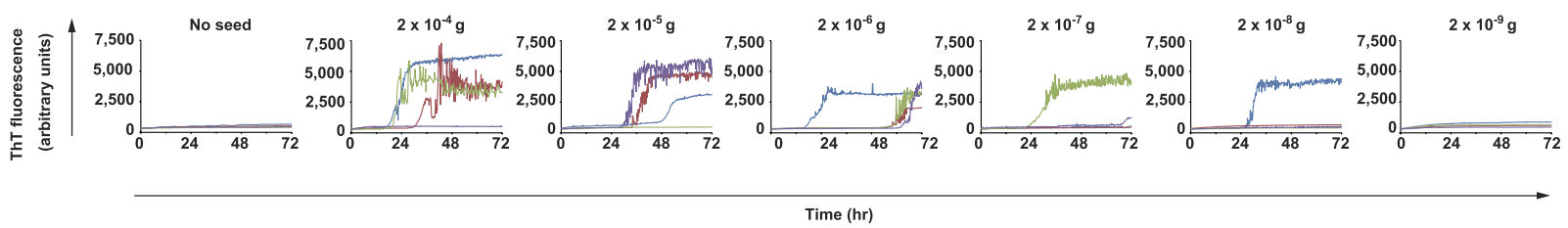

Figure 1. Stainless steel-wire with small amounts of prion seed attached can be detected by RT-QuIC reaction (wire-QuIC). The limiting dilution of $263 \mathrm{~K}-\mathrm{BH}$ was used for wire-QuIC reaction. Fibril formations of recombinant $\operatorname{PrP}$ were visualised by measurement of ThT fluorescence.

Infectious agents are highly resistant to routine decontamination methods ${ }^{13}$. High concentrations of sodium hydroxide, sodium hypochlorite or prolonged steam sterilisation are recommended methods for prion disinfection; however, most methods damage the surgical instruments ${ }^{14-17}$. Therefore, the development of new disinfection methods is needed for the safe handling and reprocessing of surgical instruments. To estimate the effectiveness of the methods, the evaluation of prion activity is of key importance.

Because of the lack of nucleic acid components, approaches for TSE rely upon methods of immunodetection including immunohistochemistry and enzyme-linked immunosorbent assay using antibodies against $\mathrm{PrP}^{18-21}$. Another evaluation method is Western blotting for protease-resistant $\mathrm{PrP}^{22}$. However, the detection range of Western blotting is narrow and not suitable to evaluate the decontamination of prion seeds. For evaluation of prion decontamination, the prion contaminated stainless steel wire test has often been used and infectivity assessed using a bioassay ${ }^{23-26}$. However, bioassays are needed for at least 1 year to quantify the infectivity, even if transgenic mice expressing $\mathrm{PrP}$ are used ${ }^{27}$. Recently, various in vitro $\mathrm{PrP}^{\mathrm{Sc}}$ formation methods were developed. We have shown that a new in vitro amplification technology called real-time quaking-induced conversion (RT-QuIC) is highly sensitive for human prion and useful for detecting small amounts of $\mathrm{PrP}^{\mathrm{Sc}}$ in cerebrospinal fluid. For the RT-QuIC reaction, intermittent shaking enhances the conversion of soluble recombinant PrP into amyloid fibrils only in the presence of $\operatorname{PrP}^{\mathrm{Sc} 28-30}$.

Here, we show that a new modified method named wire-QuIC can be applied for the direct evaluation of prion activity. Prion seeds 263K and sCJDs could firmly bind to stainless steel wire and gave rise to QuIC-positive reactions. Moreover, we demonstrated that treatment of wire with $1 \mathrm{~mol} / \mathrm{L} \mathrm{NaOH}$ solution was suitable for decontamination of prions. These results indicate that wire-QuIC can be useful to evaluate the decontamination of human prions on medical devices such as surgical instruments.

\section{Results}

To determine whether stainless steel wire is viable and does not affect amyloid formation, we conducted RT-QuIC using prion-seed-contaminated wire (wire-QuIC) instead of liquid brain homogenates (BH). To compare the efficiency of wire-QuIC, the classical RT-QuIC reaction with liquid BH was performed in parallel. As shown in Supplementary Fig. S1, the RT-QuIC reaction can detect prion seeding activities in more than $10^{-8} \mathrm{~g}$ of $\mathrm{BH}$. Importantly, the QuIC signal could also detect prion seeds attached to the wire (Fig. 1). Wire-QuIC could detect prion seeds in more than $2 \times 10^{-8} \mathrm{~g}$ of $263 \mathrm{~K}-\mathrm{BH}$.

To determine whether the wire-QuIC reaction was useful to evaluate the decontamination rate of prion from instruments such as medical equipment by washing procedures, wires with attached $263 \mathrm{~K}$ - $\mathrm{BH}$ were treated with two prion-inactivation procedures $(1 \mathrm{~mol} / \mathrm{L}$ of $\mathrm{NaOH}$ solution for $2 \mathrm{~h}$ and $3 \%(\mathrm{w} / \mathrm{v})$ sodium dodecyl sulfate (SDS) solution at $100^{\circ} \mathrm{C}$ for $10 \mathrm{~min}$ ). The positive signal of $263 \mathrm{~K}$-prion-seeds was lost after treatment with $1 \mathrm{~mol} / \mathrm{L}$ of $\mathrm{NaOH}$ solution (Fig. 2b). In contrast, no significant deletion of signal was obtained from wire-QuIC-reaction incubated with SDS solution (Fig. 2c). The same results were obtained using the classical method of RT-QuIC with liquid BHs (Supplementary Fig. S1). Although NaOH treatment removed the positive signal of RT-QuIC, there was no significant change following incubation with SDS solution.

To determine whether wire-QuIC reaction can also detect human prion, we used wire-QuIC reaction with sCJD patient $\mathrm{BH}$. The positive signal of sCJD-prion-seeds was detected in more than $10^{-11} \mathrm{~g}$ of brain using the classical method of RT-QuIC with liquid BHs (Fig. 3a). The relative concentration of prion-seeding activity, which is the number of seeding doses giving $50 \%$ positive replicate reactions $\left(\mathrm{SD}_{50}\right)$ per unit of tissue, as determined by end-point dilution RT-QuIC was $10^{10.5} \mathrm{SD}_{50} / \mathrm{g}$ brain (Fig. 3b) ${ }^{28}$. In accord with the results of $263 \mathrm{~K}-\mathrm{BHs}$ (Fig. 1), wire-QuIC reaction with sCJD-BH could detect prion seeding activities (Fig. 3c). However, the wire-QuIC reaction had a lower sensitivity than the RT-QuIC reaction, and could detect more than $10^{2.8} \mathrm{SD}_{50}$. Importantly, there was no signal in wire-QuIC with a wire that attached high concentrations $\left(10^{6.8} \mathrm{SD}_{50}\right)$ of sCJD-BH (Fig. $3 \mathrm{c}$ ).

For decontamination rate experiments, where sCJD-BH was treated with $\mathrm{NaOH}$ or SDS solution, the residuals of prion-seeds were tested by RT-QuIC and wire-QuIC. In accord with results of $263 \mathrm{~K}-\mathrm{BHs}$, in the RT-QuIC reaction, sCJD prion seeds were inactivated after $\mathrm{NaOH}$ treatment (Fig. 4b), but partial inactivation was obtained from treatments with SDS solution (Fig. 4c). The same results were obtained in wire-QuIC experiments (Fig. 5). Importantly, wires contaminated with high concentrations of sCJD-BH $\left(10^{6.8} \mathrm{SD}_{50}\right)$ were QuIC-positive when treated with SDS (Fig. 5c). This phenomenon may be because excessive dirt came off or detergents stabilised the prion-seed structure. These results suggest that the wire-QuIC reaction is useful for evaluating human sCJD prion-seed decontamination.

We developed a new washing procedure for rigid endoscopes for which electrolysis water and sonication are used. To test the decontaminating efficiency of human prion, wires that attached 10 -fold diluted sCJD-BH were 


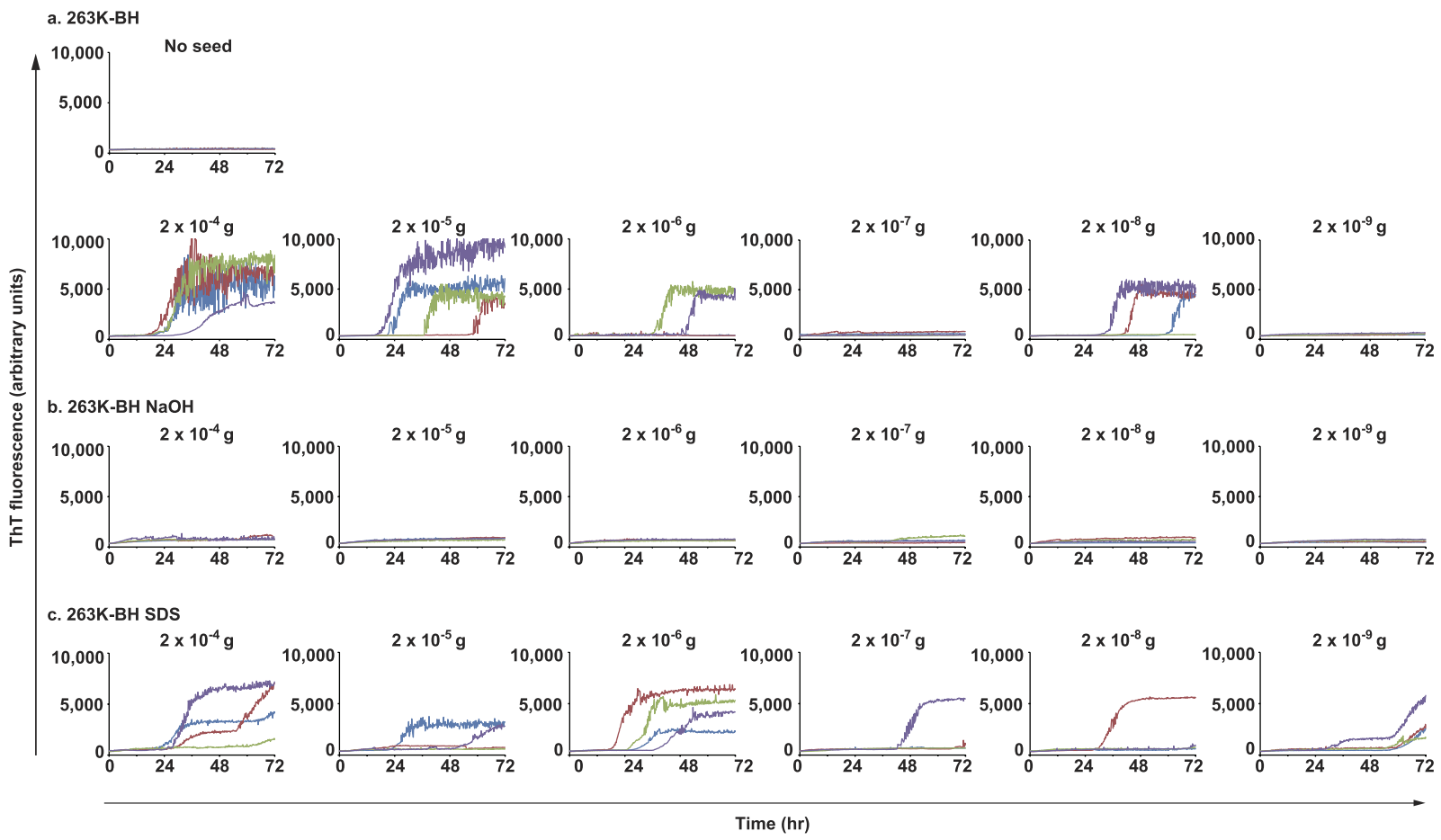

Figure 2. Wire-QuIC reaction can be useful to evaluate the decontamination rate of 263K hamster prions. Decontamination of prion $263 \mathrm{~K}$ treated with $\mathrm{NaOH}$ or SDS was evaluated by wire-QuIC reaction. Dilutions of $263 \mathrm{~K}-\mathrm{BH}$ (10-fold) were attached to wires, and then were treated with $1 \mathrm{~mol} / \mathrm{L}$ of $\mathrm{NaOH}$ for $2 \mathrm{~h}$ (b) or $3 \%$ $(\mathrm{w} / \mathrm{v}) \mathrm{SDS}$ at $100^{\circ} \mathrm{C}$ for $10 \mathrm{~min}$ (c). Wire-QuIC reactions were performed to measure the residual prion seeds to evaluate the decontamination rates of prion.

washed as described in "Materials and Methods" in $1.5 \mathrm{~mL}$ tubes, and then evaluated using the wire-QuIC reaction. No positive signal was detected with the wire-QUIC reaction (Fig. 6).

\section{Discussion}

A basic problem to prevent iatrogenic transmission of prion diseases is the lack of a convenient system to detect infectious prion on surgical instruments. In this study, we modified RT-QuIC to evaluate the residual prion seeds on wires (wire-QUIC). Although normal RT-QuIC is more suitable for and more sensitive in detect prion in cerebrospinal fluid or the brain, the wire-QuIC can detect dried prion seeds attached to wire. Both $263 \mathrm{~K}$-prion (Fig. 1) and SCJD prion (Fig. 3) on wire was amplified in vitro. This finding is in agreement with other studies showing that stainless steel wire can bind prion seeds firmly, and that surface-bound prions can transmit scrapie to recipient mice ${ }^{23,24}$. Notably, there was no signal in QuIC with high concentrations of sCJD-BH attached to the wire (Fig. 3c), and no seeding activity was observed at $2 \times 10^{-7} \mathrm{~g}$ brain dilution, while it was present at $2 \times 10^{-8} \mathrm{~g}$ (Fig. 2a). These paradoxical results may reflect the fact that the QuIC reaction is extremely sensitive and may be influenced by unknown inhibitory factors such as blood, salts or lipids.

Previous transmission studies have evaluated the prion decontamination process of wire $e^{25,26,31-34}$. Among the inactivating methods for prion, we selected two commonly used inactivation methods, treatment with $\mathrm{NaOH}$ $(1 \mathrm{~mol} / \mathrm{L} \mathrm{NaOH}$ for $2 \mathrm{~h})$ or SDS solution $\left(3 \%(\mathrm{w} / \mathrm{v}) \mathrm{SDS}\right.$ at $100^{\circ} \mathrm{C}$ for $\left.10 \mathrm{~min}\right)$, to see whether the wire-QuIC can be used for quantitative evaluation. Similar to bioassay studies ${ }^{25,26}$, the positive signals of wire-QuIC with prion seeds disappeared after treatment with $\mathrm{NaOH}$. However, SDS treatment had no significant effect. A previous study reported that a mouse adapted human prion strain, Fukuoka-1, could be completely inactivated by boiling with $3 \%(\mathrm{w} / \mathrm{v})$ SDS for at least $3 \mathrm{~min}^{35}$. Three reasons may explain this controversial result. (1) Seeding activity is not equal to infectivity. According to a previous report using $263 \mathrm{~K}$ scrapie, the $\mathrm{LD}_{50}$ of the hamster brain was approximately $10-50$-fold lower than the $\mathrm{SD}_{50}{ }^{28}$. (2) One hundred degrees Celsius is not enough for SDS inactivation, and "boiling" is an important factor. We cannot exclude this possibility without direct evaluation of these two conditions. (3) Each prion strain has a different sensitivity against SDS. We used hamster $263 \mathrm{~K}$ or human sCJD for evaluation instead of the mouse-adapted Fukuoka-1 strain. Lemmer et al. also indicated that 5\% (w/v) SDS treatment at $90^{\circ} \mathrm{C}$ could not inactivate $263 \mathrm{~K}$-prion ${ }^{26}$. Other groups also showed that rodent adapted prions have a different sensitivity to SDS compared with naturally developed original prions ${ }^{36}$.

We also tested a new washing procedure designed for endoscopes using wire-QuIC. This new procedure washes the objects with electrolysed water in combination with sonication to remove organic substances and to inactivate microorganisms and viruses (unpublished). As shown in Fig. 6, this washing procedure can decontaminate prion pathogens completely from wire. High concentrations of sodium hydroxide, sodium hypochlorite or prolonged steam sterilisation are known to be effective against prion. However, some instruments, such as flexible endoscopes, cannot withstand the heat and high concentrations of disinfectants, resulting in the discarding of 
a

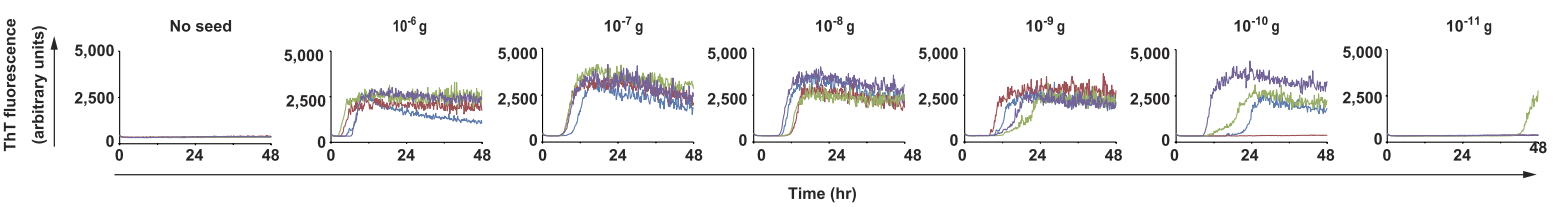

b

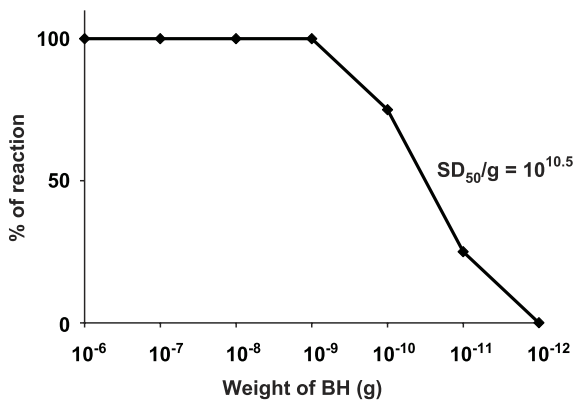

C

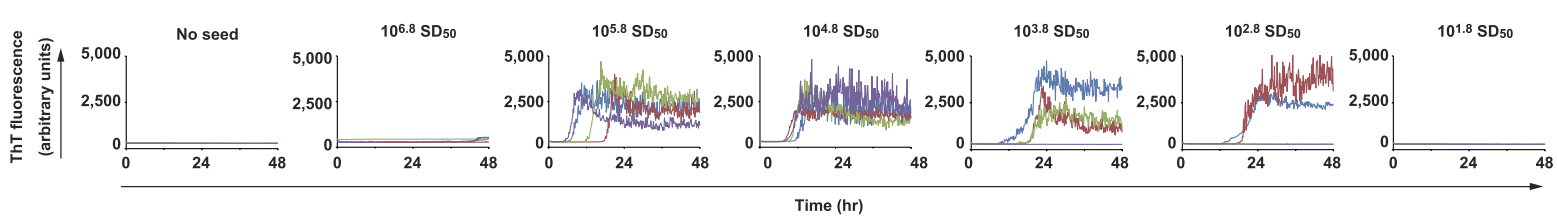

Figure 3. Wire-QuIC reaction can detect human prion seeds from contaminated stainless steel wires.

(a) Limiting dilution of sCJD-BH was used for RT-QuIC reaction. Fibril formations of recombinant PrP were visualised by measurement of ThT fluorescence. (b) Curve of seeding dose activities. Seeding dose $50 \%\left(\mathrm{SD}_{50}\right)$ per gram was $10^{10.5}$. (c) Limiting dilution of sCJD-BH was used for wire-QuIC reaction.

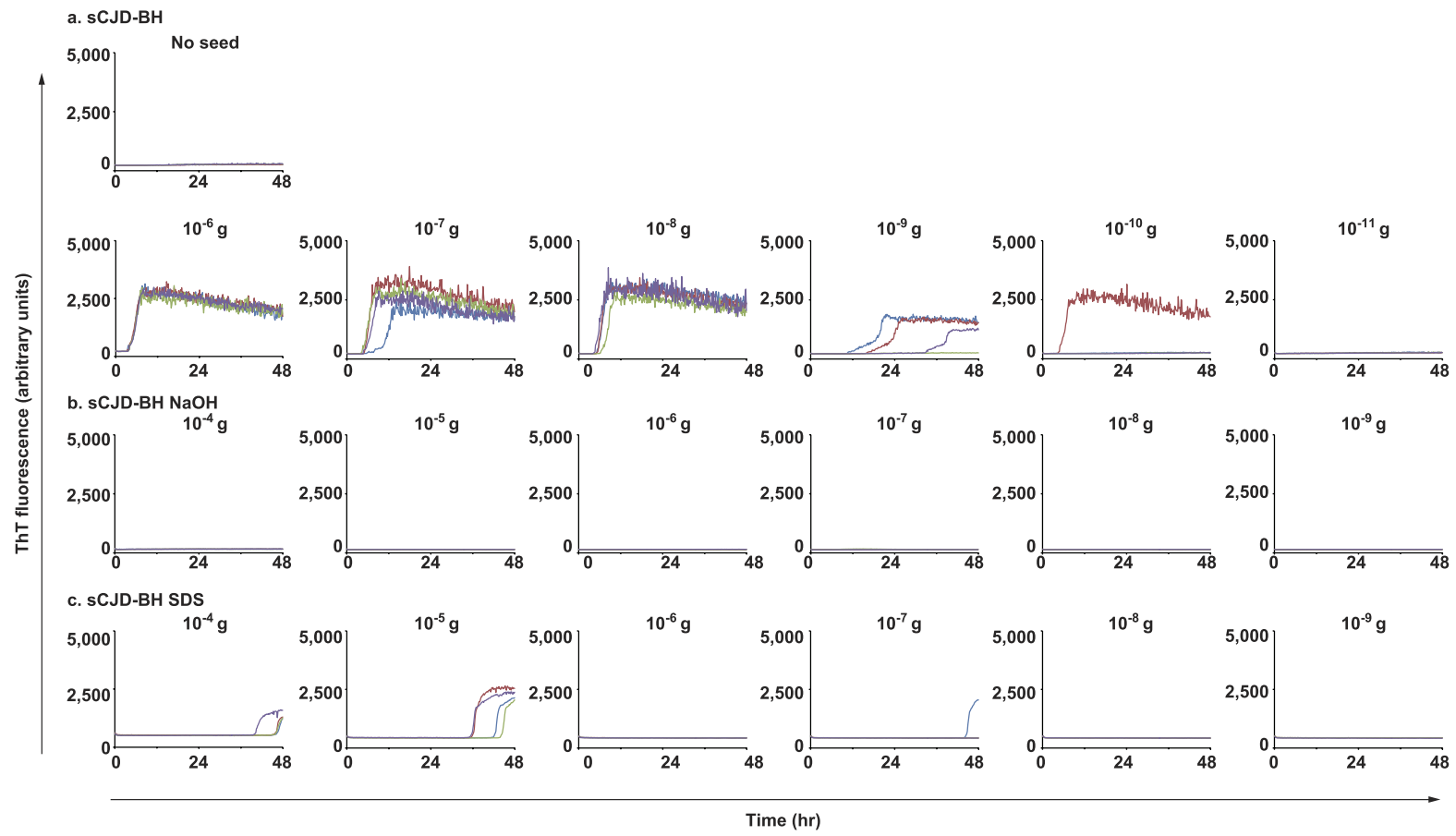

Figure 4. Inactivation of human prion seeds treated with $\mathrm{NaOH}$ or SDS solution, as evaluated by RT-QuIC reactions. Dilutions of sCJD-BH (10-fold) were treated with $1 \mathrm{~mol} / \mathrm{L} \mathrm{NaOH}$ solution for $2 \mathrm{~h}(\mathrm{~b})$ or $3 \%(\mathrm{w} / \mathrm{v})$ SDS solution at $100^{\circ} \mathrm{C}$ for $10 \mathrm{~min}(\mathrm{c})$. RT-QuIC reactions were performed to measure the residual prion-seeding activities. 


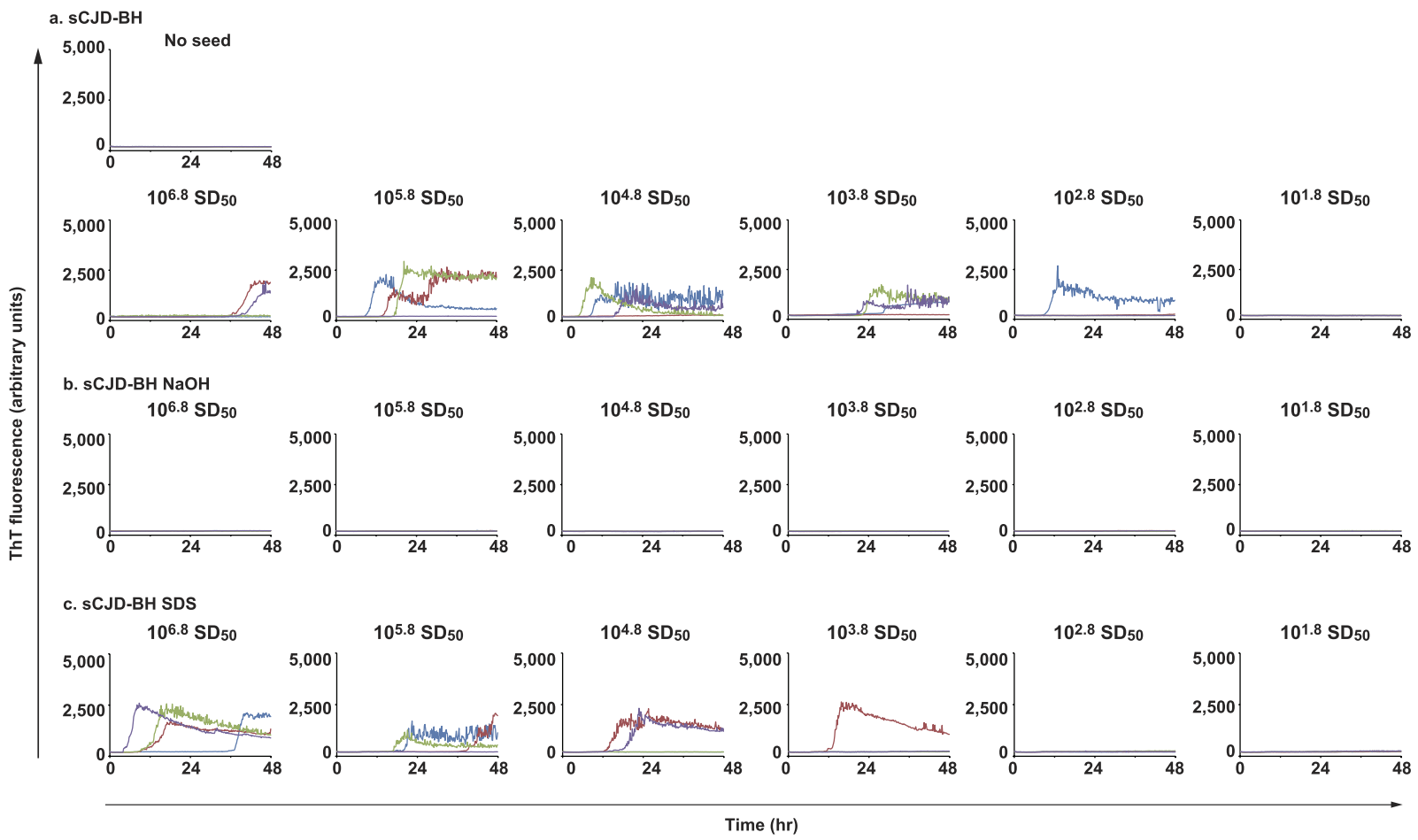

Figure 5. Wire-QuIC reaction can be useful to evaluate the decontamination rate of human prions.

Decontamination of human SCJD prion with $\mathrm{NaOH}$ or SDS were evaluated by wire-QuIC reactions. Dilutions of sCJD-BH (10-fold) were attached to wires and then were treated with $1 \mathrm{~mol} / \mathrm{L}$ of $\mathrm{NaOH}$ solution for $2 \mathrm{~h}$ (b) or $3 \%(\mathrm{w} / \mathrm{v}) \mathrm{SDS}$ solution at $100^{\circ} \mathrm{C}$ for $10 \mathrm{~min}(\mathrm{c})$. Wire-QuIC reactions were performed to measure the residual prion seeds to evaluate the decontamination rates of prion.

a. Negative control

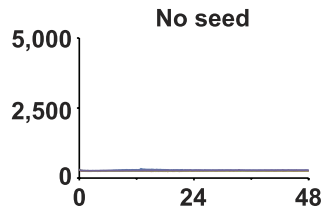

b. Unwashed
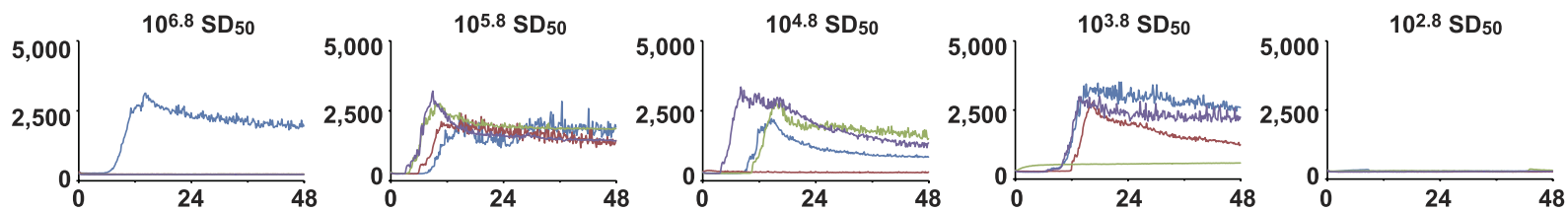

c. After wash
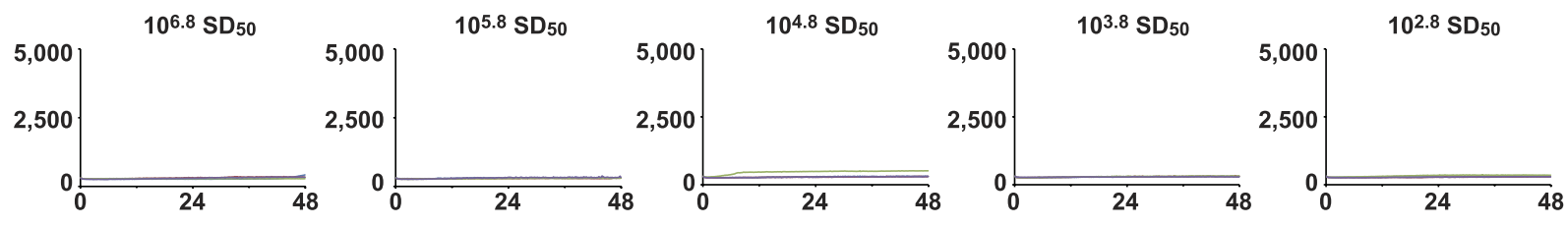

Time (hr)

Figure 6. Evaluation of cleaning using the new washing procedure by wire-QuIC reaction. Dilutions of sCJD-BH (10-fold) were attached to wires and then washed using the new procedure described in "Materials and Methods." Wire-QuIC reactions were performed to measure residual prion seeds. 
instruments after use in patients with $\mathrm{CJD}^{17}$. Therefore, this new washing procedure will reduce the risk of accidental transmission of prion. However, experiments need to be substantiated by transmission experimental data because the negative RT-QuIC reaction does not necessarily exclude the presence of infectivity on the instrument.

Taken together, the present study indicates that wire-QuIC is a useful method to evaluate washing procedures for prion contamination; however, further studies are needed in order to determine the quantitative relationship between QuIC positivity and infectivity of human prions.

\section{Methods}

Recombinant prion protein. Recombinant prion protein (recPrP) from Syrian hamster (recShaPrP23-231) or human (recHuPrP23-231) construct were expressed in Escherichia coli strain BL21 (DE3) (Stratagene, La Jolla, CA, USA) and purified as previously described ${ }^{29}$. Concentrations of recPrPs were determined by measuring the absorbance at $280 \mathrm{~nm}$. After purification, aliquots of proteins were stored at $-80^{\circ} \mathrm{C}$ in distilled water.

Preparation of brain tissue. For hamster prion (263K), brain tissues from Syrian golden hamsters infected with scrapie strain $263 \mathrm{~K}$ were collected following euthanisation at the clinical stage of disease. Animal care and experimental procedures were performed in accordance with the Regulations and Guidelines for Animal Experimentation of Nagasaki University, reviewed by the Institutional Animal Care and Use Committee of Nagasaki University and approved by the president of Nagasaki University (ID: 1107040937). For human prion, brain tissue from a human prion disease $(\mathrm{sCJD})$ patient was obtained for use in this study. Written informed consent to participate in the study was given by the patient's family. The protocol for investigation was approved by the Ethics Committee of Nagasaki University Hospital (ID: 10042823), and the study was registered with the University Hospital Medical Information Network (ID: UMIN000003301). The methods were carried out in accordance with the approved guidelines. BH in phosphate-buffered saline (PBS) were prepared (10\% w/v) using a multi-bead shocker (Yasui Kikai, Osaka, Japan). After centrifugation at 2,000 g for $2 \mathrm{~min}$, supernatants were collected and stored at $-80^{\circ} \mathrm{C}$. Dilutions of $\mathrm{BH}$ were carried out in PBS immediately prior to the reactions. For Wire-QuIC reaction, stainless steel wires (SUS304, RKC Instrument Inc., Kanagawa, Japan; diameter 0.2 mm) were cut into 5-mm-long pieces. In order to contaminate wires with $\mathrm{BH}$ in vitro, wires were incubated with several concentrations of $\mathrm{BH}$ and air-dried at room temperature for 1 day in a Petri dish.

Real-time quaking-induced conversion reaction (RT-QuIC). RT-QuIC was performed as previously described ${ }^{30}$. Briefly, $95 \mu \mathrm{L}$ of reaction buffer $(50 \mathrm{mM}$ PIPES pH 7.0,500 mM NaCl, $1 \mathrm{mM}$ EDTA and $10 \mu \mathrm{M}$ Thioflavin T (ThT) including $80 \mu \mathrm{g} / \mathrm{mL}$ of recHamPrP23-231 for $263 \mathrm{~K}-\mathrm{BH}$, or $100 \mu \mathrm{g} / \mathrm{mL}$ of recHuPrP23-231 for sCJD-BH) were loaded into wells of a 96-well optical-bottom black plate (Thermo Fisher Scientific 265301, MA, USA). Diluted BH ( $5 \mu \mathrm{L})$ was used for seeding. For the wire-QuIC reaction, air-dried wire was used. Ninety-six-well plates were covered with sealing tape (Greiner bio-one 676060, Frickenhausen, Germany) and incubated at $37^{\circ} \mathrm{C}$ in a plate reader (Infinite F200 PRO fluorescence plate reader; Tecan, Zurich, Switzerland) with intermittent shaking, consisting of shaking ( $432 \mathrm{rpm}$ orbital) for $30 \mathrm{sec}$ and no shaking for $30 \mathrm{sec}$, with a 2-min pause to measure the fluorescence. ThT fluorescence measurements were taken every $10 \mathrm{~min}$ at $440 \mathrm{~nm}$ excitation and $485 \mathrm{~nm}$ emission wavelengths. Four replicates of each diluted sample were measured. Each curve represents a single well.

Calculation of seeding dose. Seeding dose $50 \%\left(\mathrm{SD}_{50}\right)$, analogous to a bioassay's lethal dose $50 \%\left(\mathrm{LD}_{50}\right)$, were calculated using the amount of BHs which cause RT-QUIC positive signal of $50 \%$ of the wells ${ }^{37}$.

Decontaminations. Air-dried wires with attached BH were incubated for decontamination in $1 \mathrm{~mol} / \mathrm{L}$ of $\mathrm{NaOH}$ solution for $2 \mathrm{~h}$ or $3 \%(\mathrm{w} / \mathrm{v})$ Sodium dodecyl sulfate (SDS) solution at $100^{\circ} \mathrm{C}$ for $10 \mathrm{~min}$. Subsequently, wires were rinsed three times in distilled water for $1 \mathrm{~min}$, and were air-dried again.

The new washing procedure for rigid endoscopes, for which electrolysis water and sonication were used, in collaboration with Kripton Co., Ltd. and Kyowakiden Industry Co., Ltd. in the project of "Program to support development of medical equipment and devices to solve unmet medical needs 2012, 2013" and "Development of Medical Device through Collaboration between Medicine and Industry 2014" under the Ministry of Economy, Trade and Industry (METI), Japan, was performed. To prevent diffusion of the pathogen, the same washing process was performed in $1.5 \mathrm{~mL}$ tubes. Electrolysed alkaline and acidic water were prepared in the electrolysis apparatus. The apparatus consists of anode and cathode plates, made of titanium and coated with platinum, that are separated by an electrolytic diaphragm (Y-9201T, Yuasa Membrane Systems Co. Ltd., Tokyo, Japan). The electrolysed water were collected in $500 \mathrm{~mL}$ bottles and used for the experiment. Our new washing procedure consisted of five processes to perform disinfection. Wires that attached $\mathrm{BH}$ were kept separately from each other in $1.5 \mathrm{~mL}$ tube, and were then pre-washed in tap water. Wires were then treated with electrolysed alkaline water while being sonicated at $45 \mathrm{kHz}$. Subsequently, wires were rinsed in water with sonication, and treated with electrolysed acidic water, followed by rinsing in tap water. Alkaline treatment and acidic water processing were performed sequentially for 3 min each.

\section{References}

1. Rutala, W. A. \& Weber, D. J. Creutzfeldt-Jakob disease: recommendations for disinfection and sterilization. Clin Infect Dis 32, 1348-1356, doi: 10.1086/319997 (2001).

2. Chesebro, B. Introduction to the transmissible spongiform encephalopathies or prion diseases. Br Med Bull 66, 1-20 (2003).

3. Brandner, S. et al. Normal host prion protein (PrPC) is required for scrapie spread within the central nervous system. Proc Natl Acad Sci USA 93, 13148-13151 (1996).

4. Bueler, H. et al. Mice devoid of PrP are resistant to scrapie. Cell 73, 1339-1347 (1993)

5. Sakaguchi, S. et al. Accumulation of proteinase K-resistant prion protein $(\mathrm{PrP})$ is restricted by the expression level of normal $\mathrm{PrP}$ in mice inoculated with a mouse-adapted strain of the Creutzfeldt-Jakob disease agent. J Virol 69, 7586-7592 (1995). 
6. Brown, P., Brandel, J. P., Preece, M. \& Sato, T. Iatrogenic Creutzfeldt-Jakob disease: the waning of an era. Neurology 67, 389-393, doi: 10.1212/01.wnl.0000231528.65069.3f (2006).

7. Gajdusek, D. C. \& Gibbs, C. J., Jr. Transmission of two subacute spongiform encephalopathies of man (Kuru and Creutzfeldt-Jakob disease) to new world monkeys. Nature 230, 588-591 (1971).

8. Hamaguchi, T. et al. Insight into the frequent occurrence of dura mater graft-associated Creutzfeldt-Jakob disease in Japan. J Neurol Neurosurg Psychiatry 84, 1171-1175, doi: 10.1136/jnnp-2012-304850 (2013).

9. Brown, P. \& Farrell, M. A practical approach to avoiding iatrogenic Creutzfeldt-Jakob disease (CJD) from invasive instruments. Infect Control Hosp Epidemiol 36, 844-848, doi: 10.1017/ice.2015.53 (2015).

10. Brown, P. et al. Human spongiform encephalopathy: the National Institutes of Health series of 300 cases of experimentally transmitted disease. Ann Neurol 35, 513-529, doi: 10.1002/ana.410350504 (1994).

11. Wadsworth, J. D. et al. Tissue distribution of protease resistant prion protein in variant Creutzfeldt-Jakob disease using a highly sensitive immunoblotting assay. Lancet 358, 171-180 (2001).

12. Peden, A. H., Ritchie, D. L., Head, M. W. \& Ironside, J. W. Detection and localization of PrPSc in the skeletal muscle of patients with variant, iatrogenic, and sporadic forms of Creutzfeldt-Jakob disease. Am J Pathol 168, 927-935, doi: 10.2353/ajpath.2006.050788 (2006).

13. Rutala, W. A. \& Weber, D. J. \& Society for Healthcare Epidemiology of, A. Guideline for disinfection and sterilization of prioncontaminated medical instruments. Infect Control Hosp Epidemiol 31, 107-117, doi: 10.1086/650197 (2010).

14. Ernst, D. R. \& Race, R. E. Comparative analysis of scrapie agent inactivation methods. J Virol Methods 41, 193-201 (1993).

15. Steelman, V. M. Creutzfeld-Jakob disease: recommendations for infection control. Am J Infect Control 22, 312-318 (1994).

16. Steelman, V. M. Prion diseases-an evidence-based protocol for infection control. AORN J 69, 946-954, 956-967 passim; quiz 968-976 (1999).

17. Antloga, K., Meszaros, J., Malchesky, P. S. \& McDonnell, G. E. Prion disease and medical devices. ASAIO J 46, S69-72 (2000).

18. Prusiner, S. B., Scott, M. R., DeArmond, S. J. \& Cohen, F. E. Prion protein biology. Cell 93, 337-348 (1998).

19. Schaller, O. et al. Validation of a western immunoblotting procedure for bovine $\operatorname{Pr} \mathrm{P}(\mathrm{Sc})$ detection and its use as a rapid surveillance method for the diagnosis of bovine spongiform encephalopathy (BSE). Acta Neuropathol 98, 437-443 (1999).

20. Nicholson, E. M., Kunkle, R. A., Hamir, A. N., Lebepe-Mazur, S. \& Orcutt, D. Detection of the disease-associated isoform of the prion protein in formalin-fixed tissues by Western blot. J Vet Diagn Invest 19, 548-552 (2007).

21. Nicholson, E. M., Greenlee, J. J. \& Hamir, A. N. PrPSc detection in formalin-fixed paraffin-embedded tissue by ELISA. BMC Res Notes 4, 432, doi: 10.1186/1756-0500-4-432 (2011).

22. Lemmer, K., Mielke, M., Pauli, G. \& Beekes, M. Decontamination of surgical instruments from prion proteins: in vitro studies on the detachment, destabilization and degradation of PrPSc bound to steel surfaces. J Gen Virol 85, 3805-3816, doi: 10.1099/vir.0.80346-0 (2004).

23. Zobeley, E., Flechsig, E., Cozzio, A., Enari, M. \& Weissmann, C. Infectivity of scrapie prions bound to a stainless steel surface. Mol Med 5, 240-243 (1999).

24. Flechsig, E. et al. Transmission of scrapie by steel-surface-bound prions. Mol Med 7, 679-684 (2001).

25. Fichet, G. et al. Novel methods for disinfection of prion-contaminated medical devices. Lancet 364, 521-526, doi: 10.1016/S01406736(04)16810-4 (2004).

26. Lemmer, K. et al. Decontamination of surgical instruments from prions. II. In vivo findings with a model system for testing the removal of scrapie infectivity from steel surfaces. J Gen Virol 89, 348-358, doi: 10.1099/vir.0.83396-0 (2008).

27. Fischer, M. et al. Prion protein (PrP) with amino-proximal deletions restoring susceptibility of PrP knockout mice to scrapie. $E M B O$ $J$ 15, 1255-1264 (1996).

28. Wilham, J. M. et al. Rapid end-point quantitation of prion seeding activity with sensitivity comparable to bioassays. PLos Pathog 6, e1001217, doi: 10.1371/journal.ppat.1001217 (2010).

29. Atarashi, R. et al. Ultrasensitive detection of scrapie prion protein using seeded conversion of recombinant prion protein. Nat Methods 4, 645-650, doi: 10.1038/nmeth1066 (2007).

30. Atarashi, R. et al. Ultrasensitive human prion detection in cerebrospinal fluid by real-time quaking-induced conversion. Nat Med 17, 175-178, doi: $10.1038 / \mathrm{nm} .2294$ (2011).

31. Lehmann, S. et al. New hospital disinfection processes for both conventional and prion infectious agents compatible with thermosensitive medical equipment. J Hosp Infect 72, 342-350, doi: 10.1016/j.jhin.2009.03.024 (2009).

32. Yan, Z. X., Stitz, L., Heeg, P., Pfaff, E. \& Roth, K. Infectivity of prion protein bound to stainless steel wires: a model for testing decontamination procedures for transmissible spongiform encephalopathies. Infect Control Hosp Epidemiol 25, 280-283, doi: $10.1086 / 502392$ (2004).

33. Fichet, G. et al. Investigations of a prion infectivity assay to evaluate methods of decontamination. J Microbiol Methods 70, 511-518, doi: 10.1016/j.mimet.2007.06.005 (2007).

34. Vadrot, C. \& Darbord, J. C. Quantitative evaluation of prion inactivation comparing steam sterilization and chemical sterilants: proposed method for test standardization. J Hosp Infect 64, 143-148, doi: 10.1016/j.jhin.2006.06.007 (2006).

35. Tateishi, J., Tashima, T. \& Kitamoto, T. Practical methods for chemical inactivation of Creutzfeldt-Jakob disease pathogen. Microbiol Immunol 35, 163-166 (1991).

36. Giles, K. et al. Resistance of bovine spongiform encephalopathy (BSE) prions to inactivation. PLos Pathog 4, e1000206, doi: 10.1371/ journal.ppat.1000206 (2008).

37. Karber, G. 50\% End point calculation. Archiv fur Expermentelle Pathologies und Pharmakologie 162, 480-483 (1931).

\section{Acknowledgements}

We thank Dr. Yuhzo Fujita from the Teraoka Seikei Geka Hospital and Dr. Hidehiro Mizusawa, Director of the National Center Hospital of Neurology and Psychiatry for expert advice. This work was supported by a grant-in aid for the "Program to Support Development of Medical Equipment and Devices to Solve Unmet Medical Needs 2012, 2013" and "Development of Medical Devices through Collaboration between Medicine and Industry 2014" under the Ministry of Economy, Trade and Industry, Japan (24-084). Furthermore, this research was partially supported by a grant-in-aid of the Research Committee of Prion Disease and Slow Virus Infection, from the Ministry of Health, Labour and Welfare of Japan; a grant-in-aid of the Research Committee of Molecular Pathogenesis and Therapies for Prion Disease and Slow Virus Infection, the Practical Research Project for Rare and Intractable Disease from Japan Agency for Medical Research and Development, AMED; a grant from Takeda Science Foundation; a grant from the Japan Intractable Disease Research Foundation; a grant-in-aid from the Tokyo Biochemical Research Foundation; a grant provided by the Ichiro Kanehara Foundation, and a grant provided by Yokoyama Foundation for Clinical Pharmacology (No. YRY1502). These funders had no role in study design, data collection and analysis, decision to publish, or preparation of the manuscript. 


\section{Author Contributions}

T.M., R.A. and N.N. designed the study and wrote the main manuscript text. T.M., K.F., H.T. and K. Sano performed the experiments. T.M., R.A. and K. Satoh analysed the data. R.A., K. Satoh, K.I., M.H. and T. Nakayama contributed reagents/materials/analysis tools. T.M., R.A., K. Satoh, T. Nakagaki., D.I. and N.N. discussed the data.

\section{Additional Information}

Supplementary information accompanies this paper at http://www.nature.com/srep

Competing financial interests: The authors declare no competing financial interests.

How to cite this article: Mori, T. et al. A direct assessment of human prion adhered to steel wire using real-time quaking-induced conversion. Sci. Rep. 6, 24993; doi: 10.1038/srep24993 (2016).

(c) (i) This work is licensed under a Creative Commons Attribution 4.0 International License. The images or other third party material in this article are included in the article's Creative Commons license, unless indicated otherwise in the credit line; if the material is not included under the Creative Commons license, users will need to obtain permission from the license holder to reproduce the material. To view a copy of this license, visit http://creativecommons.org/licenses/by/4.0/ 